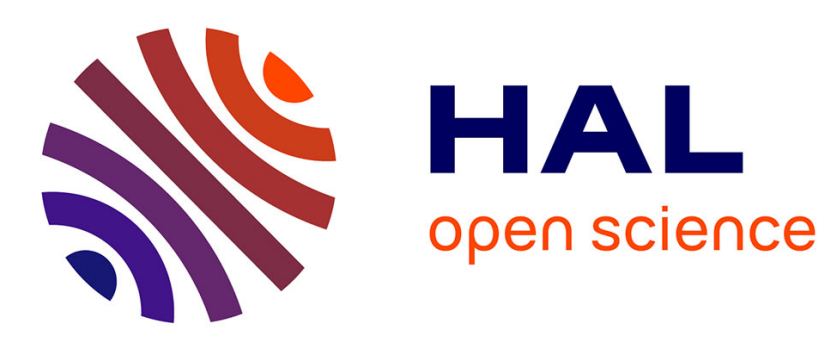

\title{
Le gouvernement de Tony Blair et la crise afghane Kenneth Owen Morgan
}

\section{To cite this version:}

Kenneth Owen Morgan. Le gouvernement de Tony Blair et la crise afghane. Observatoire de la société britannique, 2007, 3, http://osb.revues.org/395. 10.4000/osb.395 . hal-01020528

\section{HAL Id: hal-01020528 \\ https://hal.uca.fr/hal-01020528}

Submitted on 8 Jul 2014

HAL is a multi-disciplinary open access archive for the deposit and dissemination of scientific research documents, whether they are published or not. The documents may come from teaching and research institutions in France or abroad, or from public or private research centers.
L'archive ouverte pluridisciplinaire HAL, est destinée au dépôt et à la diffusion de documents scientifiques de niveau recherche, publiés ou non, émanant des établissements d'enseignement et de recherche français ou étrangers, des laboratoires publics ou privés. 


\title{
Observatoire de la société britannique
}

3 (2007)

Les années Blair

K. O. Morgan

\section{Le gouvernement de Tony Blair et la crise afghane}

\begin{abstract}
Avertissement
Le contenu de ce site relève de la législation française sur la propriété intellectuelle et est la propriété exclusive de l'éditeur.

Les œuvres figurant sur ce site peuvent être consultées et reproduites sur un support papier ou numérique sous réserve qu'elles soient strictement réservées à un usage soit personnel, soit scientifique ou pédagogique excluant toute exploitation commerciale. La reproduction devra obligatoirement mentionner l'éditeur, le nom de la revue, l'auteur et la référence du document.

Toute autre reproduction est interdite sauf accord préalable de l'éditeur, en dehors des cas prévus par la législation en vigueur en France.
\end{abstract}

revues.org

Revues.org est un portail de revues en sciences humaines et sociales développé par le Cléo, Centre pour l'édition électronique ouverte (CNRS, EHESS, UP, UAPV).

\author{
Référence électronique \\ K. O. Morgan, «Le gouvernement de Tony Blair et la crise afghane », Observatoire de la société britannique [En \\ ligne], 3 | 2007, mis en ligne le 13 mai 2011, consulté le 17 juin 2014. URL : http://osb.revues.org/395 ; DOI : \\ $10.4000 / 0 s b .395$ \\ Éditeur : Université du Sud-Toulon-Var \\ http://osb.revues.org \\ http://www.revues.org \\ Document accessible en ligne sur : \\ http://osb.revues.org/395 \\ Document généré automatiquement le 17 juin 2014. La pagination ne correspond pas à la pagination de l'édition \\ papier. \\ Tous droits réservés
}




\section{K. O. Morgan}

\section{Le gouvernement de Tony Blair et la crise afghane}

Traduction de Valérie Auda-André, Jean-Philippe Fons, Gilles Leydier, Jean-Paul Révauger et Timothy Whitton

Le 8 juin 2001, le gouvernement de Tony Blair aurait dû être au comble du bonheur ; il venait de remporter les élections pour la deuxième fois consécutive. Pour la première fois de son histoire vieille d'un siècle, le Parti travailliste avait gagné de façon exemplaire le droit d'exercer un deuxième mandat électoral. Sa majorité de 167 sièges était presque aussi écrasante que celle obtenue lors des élections de 1997. Pratiquement toutes les avancées réussies alors avaient été consolidées dans tout le pays. Le Parti conservateur était pratiquement en déroute et malgré tous les efforts déployés pendant la campagne électorale de 2001, il n'avait réussi à emporter qu'un total de 166 sièges, tout juste un de plus qu'en 1997. Le chef du Parti, William Hague, démissionna sur le champ.

Et pourtant, le 8 juin 2001, l'humeur des Travaillistes est à la réserve, presque à la dépression. Point de triomphalisme, à peine affichent-ils leur plaisir. Dans les rangs des ministres travaillistes, en commençant par Blair lui-même, on parle d'un taux de participation de $59 \%$, le plus bas jamais enregistré (11\% de moins que le faible taux relevé en 1997), de l'apathie qui frappe l'électorat et tout particulièrement les jeunes. D'un air résolu, Blair évoque la nécessité de faire comprendre que cette fois-ci le Parti honorera ses engagements surtout dans les domaines de la santé, de l'éducation et des transports. De plus, les premières semaines après les élections sont catastrophiques ; une kyrielle d'erreurs politiques donne l'impression d'un gouvernement dépourvu de réelle orientation politique ainsi que de cause en laquelle il peut croire. Se profile aussi le spectre de la contestation syndicale au sujet d'un partenariat public-privé dans certains services clés, particulièrement mal conçu - il faut le dire - par le gouvernement lui-même. A la fin du mois de juillet les ministres travaillistes n'ont qu'une seule idée en tête, partir en vacances au plus vite. Mais vient alors s'ajouter à leur malheur l'épizootie de fièvre aphteuse qui frappe le bétail et oblige bon nombre d'entre eux à passer leurs vacances d'été non pas dans les lieux de villégiature qu'affectionne le New Labour tels que la Toscane ou la Dordogne, mais dans le vent et sous la pluie de la Grande-Bretagne. Ce gouvernement, victorieux mais si défaitiste, avait donc grand besoin d'un nouveau stimulus. Comme le monde entier le sait, c'est de la manière la plus tragique que cela se produisit le 11 septembre. Depuis les attaques terroristes à New York et à Washington, la Grande-Bretagne, les travaillistes et surtout Tony Blair sont transformés. Blair lui-même a rajeuni. Lors du congrès de la Confédération Syndicale qui se tint plus tard cette même journée, Tony Blair jeta aux orties son discours de soutien aux questions de politique intérieure et déclara avec force la détermination de la Grande-Bretagne à fournir un appui sans faille aux Etats-Unis dans la lutte contre le terrorisme international. Depuis lors, et après une série très médiatisée de missions diplomatiques de part le monde, Tony Blair semble dominer à la fois la scène politique britannique et internationale. Son discours remarquable devant le Congrès du Parti travailliste réuni à Brighton - auquel j'ai pu assister - fut présidentiel. Son style oscilla entre celui de Winston Churchill et celui de William Gladstone, entre les propos charismatiques d'un chef de guerre et ceux d'un évangéliste moralisateur (celui-ci correspond sans doute bien plus à sa vraie nature). Il sera désormais nécessaire de réécrire en grande partie l'histoire du gouvernement de Tony Blair. Il est par conséquent impérieux - puisqu'il s'agit d'un élément clé qui explique bon nombre de choses - d'évaluer l'impact que pourra avoir la crise afghane sur le New Labour, sur ses ministres, son dirigeant et la mission dont le Parti serait investi. Les choses ne seront vraisemblablement plus jamais pareilles. 
Parti travailliste divisé et malheureux. Ceci est en partie dû à sa tradition séculaire de pacifisme dont l'origine remonte aux pères fondateurs tel Keir Hardie. On peut également l'attribuer à la prédilection travailliste pour les réformes concernant la politique intérieure. Ou encore s'agit-il des craintes du Parti quant au sort réservé aux libertés individuelles en temps de guerre ainsi que de son inquiétude au sujet de la psychologie irrationnelle qu'inspire le nationalisme excessif généré par les guerres et que la droite capitaliste peut exploiter.

La Première Guerre Mondiale provoqua des divisions profondes au sein des rangs des travaillistes et les socialistes opposés à la guerre tels que Hardie MacDonald et Snowden furent un certain lapse de temps écartés. Dans les années trente eurent lieu de très importantes disputes au sujet des politiques d'apaisement et de réarmement dont le symbole fut l'attaque acerbe de Ernest Bevin lors du Congrès du Parti en 1935, contre le chef de file âgé des pacifistes, George Lansbury. Après 1945, d'autres conflits furent à l'origine de divisions internes - la guerre de Corée (Aneurin Bevan et Harold Wilson démissionnent du gouvernement en 1951 pour protester contre l'importante augmentation du budget affecté au réarmement du pays) la crise de Suez (la situation était d'autant plus compliquée que le gouvernement français de l'époque était dirigé par les socialistes tels que Guy Mollet et Christian Pineau) - l'attitude de la Grande-Bretagne face à l'engagement américain au Vietnam et même lors de la guerre des Malouines lorsque le conflit fut marqué par le triomphalisme de Mme Thatcher (Réjouissezvous ! Réjouissez-vous ! proclama-t-elle). Serait-on fondé à croire, donc, que les conséquences seront différentes pour le Parti travailliste à l'occasion d'une autre guerre en 2001 ?

Tout d'abord, il conviendrait de revenir sur les raisons qui ont conduit au succès triomphal des travaillistes en 1997. Dans quelle mesure seront-ils touchés par la crise afghane ? Il y a de fortes chances pour qu'un facteur demeure inchangé, à savoir la totale inefficacité de l'opposition conservatrice ! Le déclin et la désintégration de ce parti depuis 1997 semblent s'inscrire dans la durée. Le consensus au sujet de l'Afghanistan qui lui a été imposé rend les choses encore plus périlleuses pour les conservateurs. Il semblerait qu'ils forment un parti politique sans aucun but apparent. Aussi étonnant que cela puisse paraître, ils se sont laissés dériver encore plus à droite et ont choisi comme chef de file Ian Duncan, homme politique peu connu et anti-européen invétéré. On le décrit comme un nouveau William Hague, le charisme en moins! Pour l'heure, les conservateurs sont absents de la scène politique. Il a été relevé que l'âge moyen des membres du parti est de 65 ans : aussi est-il difficile de parler d'un parti qui incarne les forces vives du pays. On évoque également la possibilité de voir les libéraux démocrates leur damer le pion dans la lutte d'occupation du terrain d'opposition face aux travaillistes même si, personnellement, j'accorde peu de crédit à cette hypothèse.

Cependant une deuxième raison qui explique le succès durable des travaillistes est peut-être amenée à disparaître. Nous avons actuellement le premier gouvernement travailliste qui n'est pas obligé de faire face à une crise économique. Ce sont les conservateurs qui désormais portent les stigmates indélébiles laissés par leur incompétence en matière de gestion des finances du pays. Il n'y a pas eu de Dunkerque financière comme ce fut le cas en 1931 (la démission après une période de forte spéculation sur la livre Sterling), en 1949 (dévaluation de la livre), en 1967 (nouvelle dévaluation) ou en 1976 (crise qui fit intervenir le Fonds Monétaire International). Depuis 1997, les choses se sont déroulées étonnamment bien. L'inflation est restée modérée (à peine $2 \%$ actuellement) et le chômage a reculé par rapport aux taux enregistrés en France ou en Allemagne. Il y a eu une forte hausse des dépenses des ménages et un pouvoir d'achat élevé a maintenu la demande des biens de consommation. Il est indéniable que la prudence dont a fait preuve Gordon Brown dans sa politique économique a été efficace même si des problèmes plus fondamentaux liés à la mauvaise gestion des entreprises, à une productivité médiocre ou à des investissements insuffisants ne sont jamais très loin. C'est là que, bien entendu, les événements en Afghanistan pourraient changer le cours des choses. Nous assistons déjà à un ralentissement durable de l'économie mondiale et les Etats-Unis se trouvent au bord de la récession. La guerre en Afghanistan a engendré de nouvelles difficultés dont des conséquences évidentes sur les industries du trafic aérien et du tourisme. Ainsi, les incertitudes de la guerre pourraient déstabiliser cet élément-clé des certitudes politiques qu'est la stabilité économique surtout en ce qui concerne les projets de relance du secteur public et 
de consolidation des services publics tels que la santé et les transports ferroviaires. Il convient de signaler la promptitude avec laquelle la bourse de Londres a su se débarrasser des effets néfastes des événements du 11 septembre et ce depuis le début. Les transactions boursières ont réagi favorablement à la diminution des taux d'intérêts et la consommation des ménages est restée forte. Cependant, il y des chances pour que l'avenir économique de la Grande-Bretagne soit moins rose, ce qui engendrera inévitablement des conséquences politiques.

La troisième raison qui peut expliquer le succès du Parti travailliste est l'absence relative de conflits sociaux. Ce phénomène pourrait justifier en partie mais de manière positive le faible taux de participation au scrutin du mois de juin 2001 tant il est vrai qu'un sentiment général de satisfaction peut engendrer une certaine apathie. Là encore la guerre en Afghanistan pour induire un changement. Tony Blair n'a pas eu à gérer la série de grèves déclenchée par les syndicats comme ce fut le cas pour les deux premiers ministres Edward Heath et James Callaghan. Il n'a pas eu non plus à faire face à la révolte contre le nouveau calcul des impôts locaux - la capitation - qui a précipité la fin du mandat de Mme Thatcher. Le seul véritable domaine où, depuis 1997, règne un sempiternel climat d'instabilité est comme d'habitude l'Irlande du Nord. Les républicains y ont commis des actes de terrorisme et les loyalistes s'en sont pris violemment aux enfants qui fréquentent les écoles catholiques. L'Assemblée, l'instance politique du pays, n'a repris ses activités qu'en octobre 2001 après avoir obtenu de la part de l'IRA un accord somme toute vague dans lequel celle-ci promettait de rendre ses importants stocks d'armes. Mais ces événements n'ont pas eu d'impact direct sur l'Angleterre, le Pays de Galles ou l'Ecosse. Le quasi effondrement du soi-disant «processus de paix » en Irlande du Nord ne les a pas affectés. L'Irlande du Nord demeure une partie unique du Royaume Uni (fort heureusement d'ailleurs) et les problèmes dont souffre le pays sont liés exclusivement à des facteurs internes, historiques et tout à fait exceptionnels. Force est de constater que l'Irlande du Nord a été le seul endroit à enregistrer un fort taux de participation au scrutin de juin 1997. Celui-ci (bien supérieur à $80 \%$ dans certaines circonscriptions électorales) témoigne de la haine ancestrale qui anime protestants et catholiques, partisans respectifs de William III et de James II en 1690.

Cela mis à part, le gouvernement de Tony Blair a exercé son mandat pendant des années de paix sociale et de satisfaction des consommateurs. La seule vraie contestation de taille est venu- et c'est étonnant, du monde rural. Aux mois de septembre et octobre 2000 il y a eu un ersatz de révolte paysanne : il s'agit d'un mélange de revendications des agriculteurs suite aux problèmes liés à la maladie de la vache folle et au déclin des prix des produits agricoles, le ressentiment des chauffeurs routiers soulevé par les prix élevés du carburant et les doléances de ceux qui pratiquent la chasse à courre qui doivent s'accommoder de l'abolition de leur macabre loisir. En bref des piquets de grève ont été dressés devant les dépôts de carburants afin d'empêcher la distribution de l'essence aux automobilistes, mais à la fin de l'an 2000, ce conflit avait trouvé une issue et dans une société urbaine - voire suburbaine - il paraît peu probable que la Grande-Bretagne rurale puisse un jour provoquer la chute du gouvernement. La guerre en Afghanistan pourrait changer cette situation à cause des quelque deux millions de Musulmans qui vivent en Grande-Bretagne dont une grande partie vient d'Asie et du souscontinent indien. Il y a eu des problèmes ethniques peu de temps après les élections dans certaines villes du nord telles que Bradford et Burnley impliquant des heurts entre des jeunes blancs et des jeunes hindous pour la plupart musulmans. Deux supporters de l'équipe de football de Leeds United sont actuellement poursuivis en justice pour le meurtre présumé d'un jeune immigré. Dans certains de ces endroits, de manière tout à fait inattendue, le fascisant Front National britannique a obtenu un très bon score aux élections. Dans nos villes du nord, les communautés musulmanes se sont souvent révélées puissantes, s'apparentant presque à des colonisateurs culturels qui d'une certaine manière se tiennent à l'écart de la société britannique. Ces communautés revendiquent leurs propres écoles et ont suscité en retour des réactions racistes de la part de la population blanche. Les relations qu'elles entretiennent avec les forces de l'ordre sont de ce fait souvent de piètre qualité. De nombreux personnages de la communauté musulmane ont contesté les bombardements américains en Afghanistan ainsi que le soutien que les britanniques y apportent. Une poignée de musulmans britanniques sont même 
partis en Afghanistan afin d'y combattre aux côtés des forces talibanes. A l'heure actuelle, les seules victimes de guerre britanniques sont du côté des talibans surtout aux alentours de Kaboul. Il est donc possible que dans l'hypothèse d'une prolongation du conflit, et a fortiori s'il doit se propager à d'autres pays musulmans tels que l'Iraq, le climat de cohésion sociale et de bonnes relations avec les minorités ethniques dont bénéficient les secteurs urbains de la Grande-Bretagne se détériore de manière significative. Pour l'instant, rien n'indique que les choses prendront cette tournure et, par le passé, la Grande-Bretagne n'a connu ni la tension qui existe entre français et communautés musulmanes (issues du continent africain bien entendu) qu'il s'agisse de la ville de Saint Denis ou celle de Marseille, ni rien de comparable avec les répercussions anti-immigrés que l'on associe à Monsieur Le Pen et ses partisans. Tony Blair s'est efforcé de souligner que ce conflit n'est pas une guerre contre l'Islam ou contre le peuple musulman et a exprimé à maintes reprises la conviction selon laquelle le Coran est un livre de paix et d'amour fraternel entre les hommes (en dépit des propos fort explicites qu'il contient sur la guerre sainte). Il serait néanmoins insensé que les historiens nient la possibilité de voir surgir de nouvelles tensions ethniques et culturelles (qui pourraient s'étendre à d'autres secteurs tels que les écoles et le logement) ce qui, par voie de conséquence, amènerait le New Labour à devoir relever de nouveaux défis.

La guerre a surtout mis en évidence l' aspect le plus marquant de l'ascension du Parti travailliste depuis 1997 : l'irrésistible domination du parti par Tony Blair lui-même. Le Parti travailliste est une coalition hybride d'éléments d'ordre politique et industriel dans laquelle jouer le rôle de dirigeant s'est toujours avéré une tâche ardue. Depuis l'époque de Keir Hardie, le Parti a toujours eu tendance à craindre les dirigeants trop puissants. La traîtrise de Ramsay Macdonald lors de son départ en 1931 pour former une alliance secrète avec les Conservateurs résonne toujours comme un avertissement. Les précédents Premiers ministres Attlee, Wilson et Callaghan ont toujours privilégié un style plus collectif. Au sein du Conseil des Ministres, Attlee fut secondé par Bevin et Morrison, Wilson par Brown et Callaghan par Healey et Foot. La domination de Tony Blair est par conséquent inédite. Sa montée en puissance a été ininterrompue depuis 1995 lorsqu'il réussit à abolir la Clause IV en faisant appel par-delà le front uni des syndicats, aux membres mêmes du Parti. Chose étonnante pour le chef de file du Parti travailliste, il tourne en ridicule le leader du Parti conservateur, John Major, en soulignant ses piètres qualités de dirigeant - « Vous suivez votre Parti tandis que moi, je dirige le mien !» déclare-t-il. Toutes les forces traditionnelles de rébellion potentielle contre la direction du Parti ont été maîtrisées par le biais de réformes internes - le Comité Central, les syndicats, le congrès du Parti ont tous été politiquement muselés. La vieille gauche se retrouve presque impuissante, même si la victoire remarquable de Ken Livingstone lors des élections municipales de Londres a démontré qu'elle a toujours du répondant si les circonstances s'y prêtent. Mais le Parti est impitoyablement mené depuis le centre par le biais des Focus Groups dont le rôle est de maintenir les liens avec les membres du Parti et également à l'aide des technologies de l'information, d'une gestion adroite des médias - rôle clé occupé par Alistair Campbell - ainsi que de dispositifs centralisés qui assurent conformité et docilité des membres. Tony Blair luimême a bénéficié d'une promotion digne d'un président sans que la moindre dissidence soit tolérée.

Ceci était vrai lorsque, entre 1994 et 1997, Tony Blair exerçait les fonctions de chef de file de l'Opposition. Il a dominé les élections de 1997. Mais ces dispositifs ont été renforcés pendant son mandat de Premier ministre et plus particulièrement depuis les élections de juin 2001. Actuellement il dirige ce que l'on pourrait presque appeler un ministère du Premier ministre après la fusion de son bureau privé avec le bureau politique. Rien qu'au 10 Downing Street, la résidence officielle du Premier ministre à Londres, plus de 200 conseillers oeuvrent directement sous la houlette de Tony Blair. En tant que Premier ministre, il a tenu de moins en moins compte du parlement et du Parti travailliste et il a contourné les structures traditionnelles fondées sur le travail des comités. Contrairement à Wilson et (surtout) Callaghan il se tient à l'écart de la salle des rafraîchissements de la Chambre des Communes. Aujourd'hui, le Conseil des Ministres s'apparente bien plus à une formalité qu'à un organe doté d'un pouvoir décisionnel : à titre d'exemple, pendant les quinze jours qui ont suivi le 11 septembre, le 
Conseil ne s'est réuni que deux fois et la durée de telles réunions n'excède pas une demie heure tout au plus. Il s'est comporté en chef d'état surtout pour des questions d'affaires étrangères, lors de la crise au Kosovo ou bien lorsqu'il a fallu prendre des sanctions à l'encontre de l'Iraq. En 1998, il s'est impliqué personnellement dans les négociations des accords du Vendredi Saint en Irlande du Nord et parfois pour régler les crises liées à la gestion du Service National de la Santé. A certains moments il a semblé agir non seulement en tant que chef du gouvernement mais en tant que chef d'état lorsque, par exemple, peu de temps après être devenu Premier ministre, il a guidé la réponse de la nation face à la mort de la Princesse Diana. Son propre pouvoir sur la machine gouvernementale est bien plus important que celui d'un président américain. Il est à n'en point douter notre premier Premier ministre post-moderne. Depuis le 11 septembre, début de la crise afghane, tout cela a été renforcé de manière substantielle. La maîtrise totale qu'exerce Tony Blair est celle d'un président. Il gouverne essentiellement par le truchement d'un Conseil de guerre restreint qui comprend un certain nombre de conseillers privés peu connus, d'anciens diplomates et d'experts en matière de défense. Il a rempli les fonctions qui d'ordinaire incombent au Ministre des Affaires Etrangères reléguant l'actuel Ministre Jack Straw et Geoff Hoon (Ministre de la Défense) à des rôles de simples figurants que l'on peut facilement écarter de la scène. Même l'omniprésence troublante de Gordon Brown, Ministre des Finances, a été marginalisée. L'envoi de troupes britanniques dans la zone de guerre n'a pas du tout été débattu en Conseil des Ministres. Vers la fin du mois de novembre, l'envoi de quelque 6000 soldats britanniques - dont la mission était d'assister à la distribution de l'aide humanitaire et de créer un environnement stable pour la mise en place d'un régime post-taliban - est le fruit non pas de décisions prises par le gouvernement britannique mais de discussions entre Tony Blair et Georges Bush. Et pourtant, à la mi-octobre, $88 \%$ de l'opinion publique britannique confirmait son soutien à la politique de Blair. Jamais sa popularité n'a semblé plus forte et jamais il n'a tenu aussi bien les rênes. Il est évident que le rôle de Blair sur la scène internationale a été considérablement valorisé grâce à ses missions diplomatiques éclair à travers le monde. En effet, sa succession très médiatisée de périples aux quatre coins du globe à coups d'avion supersonique l'a conduit à Washington, Ottawa, Paris, Bruxelles, Berlin, Moscou, Jérusalem, Amman, Riyad, Damas, Karachi et New Delhi. Ses escales n'ont pas toujours été couronnées de succès : Assad, le Président syrien l'a sermonné et en Arabie Saoudite on lui a un peu tourné le dos. En GrandeBretagne, certains de ses détracteurs ont dit qu'il ferait mieux de consacrer plus de temps aux affaires de son propre pays mais dans l'ensemble, les retombées de son action lui sont extrêmement favorables. Aux Etats-Unis, il a parfois semblé reléguer Bush au second plan à tel point que de nombreux américains ont déclaré qu'ils préféreraient avoir Blair comme président. Au Sénat américain, on l'a acclamé comme «l'un des leurs».

Pour l'instant, Blair est parvenu à un niveau d'autorité sans précédent en Grande-Bretagne depuis David Lloyd George en 1919 lors de la signature du traité de paix à Versailles. En comparaison Churchill était en 1940 un parlementaire traditionnel (dont la défaite en 1945 a dû être sans aucun doute une humiliation), tandis que les choix politiques de Mme Thatcher durant ses onze années de pouvoir ont constamment fait l'objet d'une contestation de tous les instants au sein de son propre parti. Jusqu'alors Blair a balayé toute querelle intestine même si ses relations avec Gordon Brown continuent d'alimenter la rumeur et les spéculations des médias. Après tout, Blair est le premier dirigeant travailliste à travers l'histoire depuis Keir Hardie jusqu'à John Smith à n'être nullement socialiste. Et pourtant il n'est confronté à aucune menace de la gauche. La vieille gauche, cauchemar des dirigeants travaillistes de Gaitskell à Kinnock, a plus ou moins disparu. Il n'y a plus de Bevan ni de Benn. La présence, lors de manifestations pacifiques pendant la crise afghane, de vieux personnages tels que Benn et Arthur Scargill ainsi que de vétérans de la Campagne pour le Désarmement Nucléaire tels que Monsignor Bruce Kent, est presque un aveu de défaite en soi - ils sont dans l'incapacité de faire mieux. La déconfiture de la vieille gauche est la conséquence d'une multiplicité de facteurs : les bouleversements sociaux qui ont affaibli le militantisme syndical et social et la fin de la guerre froide depuis 1989. Mais le charisme personnel de Tony Blair a également son importance. 
Cette situation charrie son lot important de dangers potentiels. Après tout, le blairisme dépend de Blair et, de ce fait, est tributaire de son propre succès. Il s'est montré particulièrement habile pour souligner les objectifs diplomatiques et humanitaires de plus grande envergure qui doivent suivre la guerre en Afghanistan. Il a déployé ces mêmes talents en exerçant une certaine pression sur Bush et ses concitoyens afin de limiter les effets de leur agressivité à l'encontre de Oussama Ben Laden (caractérisée par une approche manichéenne « Mort ou Vif ») et du réseau terroriste al-Qaïda en Afghanistan et peut-être en Irak. Autre exemple encore lorsque Blair a nommé Claire Short, cette dissidente de la vieille gauche et Ministre du Développement des Territoires d'Outre-Mer, membre de son Conseil de Guerre. Mais quelles pourraient être les conséquences si la guerre se prolongeait sans la perspective d'une solution politique et pendant que se déroulerait une catastrophe humanitaire ? Que se passerait-t-il s'il se produisait des attentats suicides sur le sol britannique ? Jusqu'alors, la position de Blair a été consolidée par la grande rapidité et le succès remarquable des forces de l'Alliance du Nord en Afghanistan. Celles-ci ont été soutenues par les bombardements américains généralement plus précis que par le passé. Mais cela ne durera pas éternellement. Bien avant les élections législatives, le style très personnel de Blair faisait de lui une cible : il y a eu le fiasco du Dôme - construction pharaonique censée marquer le passage au vingt-et-unième siècle - le scandale provoqué par Peter Mandelson, la nomination de certains de ses « copains » à la Chambre des Lords et l'hostilité même de la part de l'apolitique Institut des Femmes. Au sein même du gouvernement, Blair n'a pas les pleins pouvoirs - pour preuve les retards accumulés par rapport à la mise ne place de l'euro et la réforme du mode de scrutin. A l'intérieur du mouvement travailliste, le Parti respecte Tony Blair mais ne l'adore pas. Après tout, c'est lui qui a réinventé le Parti travailliste un peu comme s'il avait réinventé la roue. Pour les camarades qui oeuvrent dans les sections politiques locales, Tony ne sera jamais «l'un des nôtres ». Actuellement, l'isolement de Blair, même à un moment de triomphe évident, comporte donc un danger. Il existe un risque important que l'hubris ne cède la place à la némésis, comme ce fut le cas dans la Grèce antique. La culture politique britannique ne sait pas tolérer le bonapartisme. Pas plus qu'elle n'a pu supporter de Gaulle, ou un succédané à la mode britannique. Les chutes de Lloyd Georges en 1922 et de Margaret Thatcher en 1990 sont autant d'avertissements pour les dirigeants trop puissants. Le retour vers un système consensuel plus équilibré s'imposera bientôt si Tony Blair ne doit pas être la victime de son instant de toute-puissance pendant la guerre en Afghanistan.

17 Par delà ces affaires plus personnelles, la guerre en Afghanistan pourrait mettre en lumière certains aspects clés de la politique où l'attitude du New Labour a été jusqu'alors ambiguë et a manqué de clarté. On peut en distinguer trois d'entre eux.

Premièrement la vision qu'a le Parti travailliste de l'Etat. Sur ce point, le New Labour n'a jamais pu se décider même si depuis 1945 au moins, le Parti travailliste a été en mesure d'apprécier le pouvoir qu'a l'Etat - et surtout le gouvernement central - d'offrir des opportunités et de dynamiser dans le but de créer une société plus juste et plus équitable. Les principaux théoriciens du Parti, de Tony Crosland à Monsieur et Madame Webb, étaient tous des étatistes. Tout comme ses prédécesseurs, le New Labour s'est également servi de l'Etat pour promouvoir les services publics et protéger les personnes vulnérables. Il a eu recours aux mesures de redistribution, notamment le crédit d'impôt ainsi que les campagnes de lutte contre la pauvreté et particulièrement celle qui touche les enfants. Il a également signé le Chapitre Social Européen qui comprend un salaire minimum. Pourtant, il s'est montré réticent, presque thatchérien, à se servir du pouvoir de l'Etat pour modifier les mécanismes du libre jeu des forces du marché. La déréglementation de l'économie et le marché de l'emploi «flexible» ont été des articles de foi pour le gouvernement centre-gauche britannique. Cela n'a jamais été le cas, par exemple, pour le président Jacques Chirac et le centre-droit français. Le Parti travailliste ne s'est servi de l'Etat que de manière restreinte pour promouvoir le bien public. Cela a été plus qu'évident dans l'éducation supérieure où la gratuité de l'enseignement à l'université ainsi que les bourses aux étudiants ont été abolies. Après la guerre en Afghanistan le débat sur l'initiative financière censée optimiser la compatibilité entre fonds privés et fonds publics pourrait constituer un élément fondamental dans la conception qu'a le Parti travailliste 
de son propre avenir. La manière dont le gouvernement de Blair pourra continuer d'attribuer des fonds plus importants aux services de santé, de l'éducation, aux transports en commun et à l'environnement en sera le point d'orgue. Si les travaillistes ne se montrent pas à la hauteur des attentes, si les entreprises telles que le métro de Londres accusent un nouveau recul, il y aura inévitablement des problèmes.

Deuxièmement se pose la question de la décentralisation. Après avoir appelé à des votes référendaires, le Parti travailliste a doté l'Ecosse d'un parlement et le Pays de Galles d'une assemblée. Il y a également une assemblée en Irlande du Nord, un maire à Londres et le vague espoir qu'une administration régionale pourra voir le jour en Angleterre. Blair déclare à juste titre avoir cédé beaucoup plus de pouvoir que n'importe quel autre Premier ministre. Pourtant, il y a eu, pour emprunter un gallicisme bien connu, du dirigisme. Au Pays de Galles et à Londres des pressions depuis le pouvoir central se sont fait sentir pour dicter le choix des dirigeants (Rhodri Morgan au Pays de Galles et Ken Livingstone à Londres) ce qui a provoqué à deux reprises dans la capitale des défaites désastreuses à la fois pour l'organisation politique du Parti et ses dirigeants. Cette politique sera mise à l'épreuve en Ecosse dotée pour la première fois d'un Premier Ministre, Jack McConnell, qui n'est pas issu de Westminster. Dans ce pays, une série de choix politiques dignes de la vieille gauche a été votée décrétant la gratuité de l'enseignement supérieur et de la couverture maladie pour les personnes âgées. Il semblerait que la culture politique en Ecosse soit bien plus radicale qu'ailleurs. La crise afghane pourrait accroître la tension au sein du Parti travailliste entre les deux courants, celui de la décentralisation et celui du dirigisme. Elle pourrait contribuer au développement d'un centralisme accru. Ce dernier s'est déjà manifesté à travers les tentatives de réduire les libertés civiques et à cet égard le projet de loi « anti-terroriste » dont les pouvoirs plus importants conférés aux forces de l'ordre et le non-respect partiel de la Convention Européenne des Droits de l'Homme ont conduit à de graves dissensions intestines. Pendant le mandat du dernier parlement et en ce qui concerne les droits civiques, le gouvernement a subi quelques défaites significatives à la Chambre des Lords- par exemple sur les restrictions imposées au jugement par jury - et il demeure vulnérable. Le nouveau Ministre de l'Intérieur, David Blunkett, n'est pas plus libertaire que son prédécesseur, Jack Straw.

En dernier lieu, il y a toujours l'Europe. Sans souffrir de l'europhobie des conservateurs, le Parti travailliste s'est montré depuis 1997 étonnamment prudent dans ce domaine et d'une façon qui transcende une quelconque rivalité entre Blair et Brown. En dépit d'une majorité de 167 sièges, un Parti conservateur désespérément faible, le soutien des Libéraux-Démocrates pro-européens et la présence de gouvernements sociaux-démocrates dans la plupart des pays européens, le Parti travailliste s'est montré malgré tout hésitant. En ce qui concerne l'implication dans l'Europe, les atermoiements du gouvernement de Blair sont ceux qui caractérisent les gouvernements britanniques depuis que celui dirigé par Attlee a refusé de se joindre au projet de Schumann à savoir la création en 1950 d'une Communauté de l'Acier et du Charbon. De la même manière le gouvernement de Eden a boudé la conférence de Messine en 1955 et les préparatifs qui devaient mener au Traité de Rome. Tout comme ses prédécesseurs, Blair hésite entre être pris dans la relation dite « spéciale » avec les Etats-Unis et occuper une place « au cœur de l'Europe » selon la formule trompeuse et très connue de John Major.

Mais sur ce point la crise afghane pourrait induire un réel changement de trajectoire. Blair manie la rhétorique transatlantique. En effet, sa façon de parler, ses goûts culturels et vestimentaires ont une résonance et une apparence américaines même si - à l'inverse de Gordon Brown - il aime passer ses vacances en France et parle même le français, une première pour un Premier ministre depuis Eden. Blair est de loin l'allié européen le plus proche de Bush et le seul a avoir proposé l'engagement d'un nombre significatif de troupes (même si la France et l'Allemagne pourraient en faire autant). Pourtant, avec le temps, il devient plus européen. La mise en place d'une approche européenne des questions de sécurité et la coordination d'une réponse transcontinentale au terrorisme le rapprochent de l'Europe. Il a profité de son discours prononcé à l'occasion de la conférence de son parti à Brighton pour formuler sa plus forte déclaration en date en faveur de l'adhésion de la Grande-Bretagne à l'euro, bien que depuis lors il y ait eu des moments d'hésitation. Le Chancelier Schröder était 
un autre personnage de la scène politique présent dans la station balnéaire de Brighton et a reçu un accueil chaleureux. Le discours de Blair devant l'Institut de la Recherche Européenne le 23 novembre (2001) a été l'occasion pour lui de faire avancer encore plus ce processus lorsqu'il a essayé de démontrer que depuis 1957 la Grande-Bretagne avait laissé passer de nombreuses occasions de s'impliquer en Europe. Les sondages d'opinion ont consolidé sa position. Ils ont montré que même si l'opinion britannique est hostile à l'euro, elle estime inévitable une adhésion éventuelle tandis que $81 \%$ des sondés considèrent que les événements du 11 septembre impliqueront une plus forte collaboration entre les partenaires européens. Il semble tout à fait possible que le referendum sur l'euro soit organisé en 2003 en admettant que les prochaines élections aient lieu en 2005. On pourrait donc assister à un engagement profondément nouveau. Il se pourrait, peu ou prou, que la crise Afghane confère à la GrandeBretagne le statut, inédit dans l'histoire du pays, de puissance européenne.

La crise afghane met en exergue l'ambiguïté fondamentale qui est au cœur du New Labour. La base de l'électorat travailliste dans le Nord de l'Angleterre, en Ecosse et au Pays de Galles a peu de choses en commun avec les élites mafieuses des quartiers chics de Londres. De nos jours le Parti travailliste est à cheval sur deux circonscriptions, non seulement d'un point de vue géographique mais également idéologique. Il existe bien des incertitudes quant à ce que représente le Parti travailliste hormis l'affirmation nébuleuse selon laquelle il serait quelque chose de «nouveau ». Il n'a aucune philosophie politique même si la Société des Fabiens s'efforce courageusement d'en fournir une. Ses tentatives pour définir les objectifs du parti se sont révélées dérisoires - pour s'en convaincre il n'est que de regarder le « mélimélo abstrait de généralités » (pour citer le biographe bienveillant de Blair, John Rentoul) dont sont composées la troisième voie et les doctrines qui la sous-tendent, ou les tentatives sans convictions faites pour trouver des âmes sœur en Australie, à Singapour ou auprès des démocrates de Clinton. Il y a un grand vide au centre du système des valeurs et des croyances du Parti travailliste. Comme à aucun autre moment de l'histoire du travaillisme britannique, le New Labour a montré son efficacité à être un parti de gouvernement et une machine merveilleuse pour gagner des voix. Mais il a besoin de tisser de nouveaux liens avec le parti et avec le pays. En France, le parti de Jospin affiche à bien des égards les mêmes caractéristiques que celui de Jaurès ou de Léon Blum. Compte tenu de la renaissance revendiquée par le New Labour, il ne peut pas y avoir ce type de qualité ancrée dans la profondeur. Attlee et Blair sont aux antipodes l'un de l'autre. De nombreux nouveaux disciples venus renforcer le New Labour sont issus du monde des affaires et de la classe moyenne de l'Angleterre «moyenne » : ils pourraient se volatiliser tout comme les Sociaux Démocrates l'ont fait pendant les années quatre-vingt (à bien des points de vue il s'agit des mêmes personnes !).

Les prochaines élections législatives en Grande-Bretagne, qui auront probablement lieu en 2005, ne seront pas gagnées dans les montagnes de l'Afghanistan ou grâce à une plateforme de guerre. Elles seront gagnées à Kettering et à Kilmarnock, pas dans la Passe de Khyber ou à Kaboul. Elles se gagneront selon la manière dont les Travaillistes auront tenu leurs engagements sociaux et économiques dans les domaines de l'économie, de la santé, de l'éducation et plus généralement dans les services publics et selon l'affection que l'opinion publique lui témoignera. La désaffection de l'électorat et le taux de participation de $59 \%$ lors des élections en 2001 sont de sérieux avertissements. On ne peut pas dire à l'heure actuelle que la population porte le gouvernement dans son cœur (ni le système politique dans son ensemble). Pendant les années Attlee, le Festival de la Grande-Bretagne sur la rive droite de la Tamise en 1951 fut le symbole d'une nation pourvue d'une conscience claire de son histoire et de son identité alors qu'elle se hissait hors d'une période traumatisante de guerre et de l'austérité qui en avait découlé. Je le sais car j'y étais, jeune lycéen de seize ans qui s'en émerveillait en goûtant aux joies de la fête foraine installée à Battersea à quelques kilomètres de là. En 1945, le manifeste électoral des travaillistes « Tournons nous vers l'avenir » incarnait ce sentiment d'espoir. En 2001, le Dôme installé à Greenwich était en comparaison sans joie et symboliquement vide, un monument à la gloire de ce qui est politiquement correct sponsorisé par McDonald, empli de bruit et de fureur sans aucune signification, pour citer Macbeth de Shakespeare. 
Il est bien évidemment certain qu'après le Festival de la Grande-Bretagne en 1951, le Parti travailliste a perdu les élections mais il a obtenu presque quatorze millions de voix (plus que les conservateurs vainqueurs, et un record avant la victoire en 1992 de John Major) c'est-àdire 48,8 \% du total. A titre de comparaison, le Parti travailliste s'est adjugé en 2001 une majorité de 167 sièges grâce à 7 millions de voix (malgré une population évidemment bien plus importante) c'est-à-dire $42 \%$ des suffrages. Même après la défaite électorale de 1951, le Parti travailliste avait toujours un sens du passé et une vision de l'avenir. En dépit de tous ses succès électoraux, le New Labour sous la direction de Tony Blair doit rapidement avancer par rapport à la guerre en Afghanistan pour renouer avec son passé en même temps qu'avec ses perspectives d'avenir s'il souhaite de nouveau se «tourner vers l'avenir » avec confiance.

\section{Pour citer cet article}

Référence électronique

K. O. Morgan, «Le gouvernement de Tony Blair et la crise afghane », Observatoire de la société britannique [En ligne], 3 I 2007, mis en ligne le 13 mai 2011, consulté le 17 juin 2014. URL : http:// osb.revues.org/395; DOI : 10.4000/osb.395

\section{$\grave{A}$ propos de l'auteur}

\section{K. O. Morgan}

FBA, Baron Morgan of Aberdyfi

Universitaire et historien, membre de la Chambre des Lords

\section{Droits d'auteur}

Tous droits réservés 